\title{
Altered Expression of RNA Splicing Proteins in Alzheimer's Disease Patients: Evidence from Two Microarray Studies
}

\author{
Jenny Wong \\ Illawarra Health and Medical Research Institute, and School of Biological Sciences, \\ University of Wollongong, Wollongong, N.S.W., Australia
}

\section{Key Words}

Alternative splicing · Microarray · Brain tissue, postmortem · Gene expression ·

Alzheimer's disease $\cdot$ Hippocampus

\section{Abstract}

Background/Aims: Dysregulation of pre-mRNA splicing from an altered expression of RNA splice-regulatory proteins may act as the convergence point underlying aberrant gene expression changes in Alzheimer's disease (AD). Methods: Two microarray datasets from a control/AD postmortem brain cohort of 31 subjects - 9 controls and 22 AD subjects (National Center for Biotechnology Information (NCBI) Gene Expression Omnibus (GEO) database) were used. Results: Between the two microarray studies, the expression of six splice-regulatory protein genes showed concordant changes in AD. These genes were then correlated with gene expression changes of transcripts reported to be altered in AD. Amyloid beta (A4) precursor protein and tropomyosin receptor kinase $B$ transcripts were found to correlate significantly with the same splice-regulatory proteins in the two studies. Conclusion: This study highlights a susceptibility network that can potentially link a number of susceptibility genes.

Copyright (C) 2013 S. Karger AG, Basel

\section{Introduction}

Alzheimer's disease (AD) is the most common form of dementia accounting for between 50 and $70 \%$ of cases globally $[1,2]$. The presence of intracellular neurofibrillary tangles, extracellular neuritic plaques, and brain-wide neurodegeneration are key pathological features which define AD [3]. Other pathological processes associated with AD include al- 
tered levels of neurotransmitter receptor expression [4] as well as dysregulation of cell signaling [5-7], inflammatory response [8, 9], synaptic transmission [10-12], and cholesterol metabolism $[13,14]$. At present, a complete understanding of the underlying causes of these pathogenic processes remains elusive. It is predicted that there is a common underlying process that is dysregulated in $\mathrm{AD}$, which serves as a convergence point that links the multitude of dysfunctional signatures.

Alternative splicing is a widespread gene-regulatory process by which exons of primary transcripts (pre-mRNAs) are spliced into different arrangements to produce structurally distinct mRNA variants. This mechanism of gene product diversification plays a critical role in controlling cellular differentiation and development in response to environmental, temporal, or cell type-specific cues $[15,16]$. It is estimated that more than $75 \%$ of genes in the human genome are alternatively spliced [17]. Dysregulation in alternative splicing has been linked to a number of human diseases including some neurodegenerative diseases (e.g. frontotemporal dementia with parkinsonism) [18]. However, in AD, few studies have investigated the link between alternative splicing dysregulation, aberrant splice-regulatory protein expression, and $\mathrm{AD}$ progression. In a recent study, it has been found that generation of the tropomyosin receptor kinase B (TrkB) alternative transcript TrkB-Shc is regulated by the serine/arginine protein Srp20, and that biochemical manipulation of its expression in neuronal cell lines and exposure of cells to amyloidogenic factors could modulate TrkB pre-mRNA splicing and TrkB-Shc expression [19]. In other dementias, alterations in the levels of splice-regulatory protein expression have been shown to affect alternative splicing in frontotemporal dementia with parkinsonism. For instance, in humans, alterations in adult-specific tau exon 10 splicing have been demonstrated to lead to abnormal ratios of tau isoform expression [20-22]. Considering that one splice-regulatory protein is capable of regulating the splicing of multiple pre-mRNAs, it is hypothesized that dysregulation of pre-mRNA splicing from the altered expression of key splice-regulatory proteins in AD may underlie the aberrant changes in gene expression in multiple AD-affected pathways during disease progression.

\section{Materials and Methods}

\section{Datasets}

The hippocampal CA1 microarray datasets derived from postmortem brain tissue from a total of 31 subjects -9 controls and 22 AD subjects - of varying AD severity ( $n=7$ incipient, $\mathrm{n}=8$ moderate, and $\mathrm{n}=7$ severe) were obtained from the National Center for Biotechnology Information (NCBI) Gene Expression Omnibus (GEO) database (Affymetrix GeneChip (HG-U133A); accession GDS810 [23] and GSE28146 [24]). Cohort demographics are listed in table 1.

To determine whether there are changes in splice-regulatory protein gene expression during AD progression, expression profiles of all genes involved in RNA splicing were collated from the GDS810 microarray. Genes that were statistically significant by one-way analysis of variance (ANOVA) with a p value $<0.05$ or showed a trend towards statistical significance with a $\mathrm{p}$ value range between 0.05 and 0.1 were then mined from the GSE28146 microarray. Considering that a specific group of genes were to be assessed, all gene expression profiles were included, even those with low expression.

\section{Statistical Analysis}

Statistical analyses were conducted using Statistica 7 (StatSoft Inc., 2000, Statistica for Windows). One-way ANOVA was conducted to assess changes in gene expression during AD 
Table 1. Cohort demographics

\begin{tabular}{|c|c|c|}
\hline & Gender & Age, years \\
\hline Control 976 & male & 85 \\
\hline Control 1003 & male & 80 \\
\hline Control 1008 & female & 92 \\
\hline Control 1012 & male & 80 \\
\hline Control 1015 & male & 75 \\
\hline Control 1018 & female & 97 \\
\hline Control 1030 & male & 95 \\
\hline Control 1039 & male & $77^{\mathrm{a}}$ \\
\hline Control 1040 & male & 87 \\
\hline Incipient 715 & female & 101 \\
\hline Incipient 720 & female & 95 \\
\hline Incipient 994 & female & 83 \\
\hline Incipient 1019 & male & 88 \\
\hline Incipient 1029 & female & 91 \\
\hline Incipient 1034 & male & 88 \\
\hline Incipient 1043 & female & 97 \\
\hline Moderate 826 & female & 85 \\
\hline Moderate 832 & female & 89 \\
\hline Moderate 856 & female & 83 \\
\hline Moderate 965 & female & 82 \\
\hline Moderate 1020 & female & 79 \\
\hline Moderate 1025 & male & 81 \\
\hline Moderate 1031 & female & 86 \\
\hline Moderate 1037 & male & 82 \\
\hline Severe 701 & male & 85 \\
\hline Severe 723 & female & 65 \\
\hline Severe 807 & male & 93 \\
\hline Severe 819 & female & 79 \\
\hline Severe 867 & female & 94 \\
\hline Severe 872 & female & 79 \\
\hline Severe 1036 & female & 93 \\
\hline
\end{tabular}

${ }^{a}$ Control 1039 is excluded in the GSE28146 microarray.

progression. ANOVAs were followed up with the Fisher LSD post hoc analysis to assess significance in gene expression between AD severity groups. Pearson's product moment correlations were used to determine whether any relationship existed between splice-regulatory protein gene expression and the expression of AD susceptibility genes. A p value $<0.05$ (twotailed) was considered statistically significant.

\section{Results}

In this current report, two publically available microarray datasets from a control/AD hippocampal brain cohort from the NCBI GEO database (Affymetrix GeneChip (HG-U133A); accession GDS810 [23] and GSE28146 [24]) were utilized. In both studies [23, 24], the expression of genes significantly altered in AD was classified into functional categories and biological pathways of the gene ontology consortium (www.geneontology.org). However, a specific category for genes involved in RNA splicing was not described and genes involved in RNA splicing were likely grouped into other gene ontology categories. Thus, to determine whether genes involved in RNA splicing are altered during AD progression, genes whose gene 
Wong: Altered Expression of RNA Splicing Proteins in Alzheimer's Disease Patients:

Evidence from Two Microarray Studies

Table 2. Microarray gene expression changes in the CA1 hippocampus ( $\mathrm{p}<0.05$; GDS810 microarray)

\begin{tabular}{|c|c|c|c|c|c|c|c|c|}
\hline Probe & Gene & Description & Control & Incipient & Moderate & Severe & $\begin{array}{l}\text { ANOVA } \\
\mathrm{F}\end{array}$ & $\begin{array}{l}\text { ANOVA } \\
\mathrm{p}\end{array}$ \\
\hline 206809_s_at & HNRNPA3 & $\begin{array}{l}\text { heterogeneous nuclear } \\
\text { ribonucleoprotein A3 }\end{array}$ & $461.2 \pm 62.2$ & $305.9 \pm 48.3$ & $362.1 \pm 53$ & $222.1 \pm 38.4$ & 3.63 & $0.03^{\mathrm{a}}$ \\
\hline 208713_at & HNRNPUL1 & $\begin{array}{l}\text { heterogeneous nuclear } \\
\text { ribonucleoprotein U-like } 1\end{array}$ & $610.9 \pm 39.4$ & $809.7 \pm 72.7$ & $757.5 \pm 38.8$ & $836.6 \pm 67$ & 3.73 & $0.02^{\mathrm{a}}$ \\
\hline 202903_at & LSM5 & $\begin{array}{l}\text { U6 snRNA-associated } \\
\text { Sm-like protein LSm5 }\end{array}$ & $108.8 \pm 10.6$ & $167.5 \pm 19.9$ & $162.5 \pm 7.9$ & $186.5 \pm 29.8$ & 3.79 & $0.02^{\mathrm{a}}$ \\
\hline 202697_at & NUDT21 & $\begin{array}{l}\text { nucleoside diphosphate-linked } \\
\text { moiety X }\end{array}$ & $135.4 \pm 10.8$ & $188.6 \pm 14.2$ & $136 \pm 14$ & $141.2 \pm 14.4$ & 3.54 & $0.03^{\mathrm{a}}$ \\
\hline 213852_at & RBM8A & RNA-binding motif protein 8A & $1,490.1 \pm 72.1$ & $1,899.7 \pm 104$ & $1,806 \pm 80.1$ & $1,887.6 \pm 173.1$ & 3.39 & $0.03^{\mathrm{a}}$ \\
\hline 216842_x_at & RBMY1J & $\begin{array}{l}\text { RNA-binding motif protein, } \\
\text { Y-linked, family } 1 \text {, member J }\end{array}$ & $144.1 \pm 19.4$ & $275.9 \pm 42.7$ & $156.5 \pm 26.7$ & $232.1 \pm 48.3$ & 3.37 & $0.03^{\mathrm{a}}$ \\
\hline 207842_s_at & CASC3 & $\begin{array}{l}\text { cancer susceptibility } \\
\text { candidate } 3\end{array}$ & $1,372.3 \pm 115.2$ & $1,646.7 \pm 183.4$ & $2,256.4 \pm 256.3$ & $2,198.7 \pm 212.3$ & 5.12 & $0.006^{\mathrm{b}}$ \\
\hline 203376_at & CDC40 & cell division cycle 40 homolog & $1,291.1 \pm 84.1$ & $1,174.3 \pm 91.9$ & $913.5 \pm 73.4$ & $1,033.8 \pm 67.7$ & 4.46 & $0.01^{\mathrm{b}}$ \\
\hline 201077_s_at & NHP2L1 & $\begin{array}{l}\text { nonhistone chromosome } \\
\text { protein } 2 \text {-like } 1\end{array}$ & $1,995.8 \pm 74.2$ & $1,764 \pm 105.5$ & $1,599.1 \pm 42.9$ & $1,487.1 \pm 107.8$ & 7.39 & $0.0009^{\mathrm{b}}$ \\
\hline 200057_s_at & NONO & $\begin{array}{l}\text { non-POU domain-containing } \\
\text { octamer binding }\end{array}$ & $3,671.8 \pm 98.9$ & $3,872.7 \pm 103.9$ & $4,017.1 \pm 164.8$ & $4,171.8 \pm 110.5$ & 3.07 & $0.04^{\mathrm{b}}$ \\
\hline 202635_s_at & POLR2K & $\begin{array}{l}\text { polymerase (RNA) II } \\
\text { (DNA-directed) polypeptide K, } \\
7.0 \mathrm{kDa}\end{array}$ & $465.9 \pm 61.3$ & $367.5 \pm 44.5$ & $253.2 \pm 22.6$ & $409.4 \pm 46.2$ & 3.85 & $0.02^{\mathrm{b}}$ \\
\hline 208652_at & PPP2CA & $\begin{array}{l}\text { serine/threonine protein } \\
\text { phosphatase } 2 \mathrm{~A} \text { catalytic } \\
\text { subunit alpha isoform }\end{array}$ & $3,154.9 \pm 298.8$ & $2,558.9 \pm 234.3$ & $2,255.6 \pm 130.1$ & $1,840.5 \pm 278.6$ & 5.15 & $0.006^{\mathrm{b}}$ \\
\hline 221547_at & PRPF18 & $\begin{array}{l}\text { PRP18 pre-mRNA processing } \\
\text { factor } 18 \text { homolog }\end{array}$ & $220 \pm 15.2$ & $239 \pm 11.9$ & $161.4 \pm 17.1$ & $258.2 \pm 21$ & 6.25 & $0.002^{\mathrm{b}}$ \\
\hline 218053_at & PRPF40A & $\begin{array}{l}\text { PRP } 40 \text { pre-mRNA processing } \\
\text { factor } 40 \text { homolog } \mathrm{A}\end{array}$ & $876 \pm 73.5$ & $979.3 \pm 63$ & $1,334.4 \pm 125.6$ & $1,193.6 \pm 79.9$ & 5.54 & $0.004^{\mathrm{b}}$ \\
\hline 211270_x_at & PTBP1 & $\begin{array}{l}\text { polypyrimidine tract-binding } \\
\text { protein } 1\end{array}$ & $565.9 \pm 50.9$ & $683.1 \pm 78.9$ & $850.1 \pm 87.4$ & $870.1 \pm 76$ & 4.1 & $0.02^{\mathrm{b}}$ \\
\hline 211271_x_at & PTBP1 & $\begin{array}{l}\text { polypyrimidine tract-binding } \\
\text { protein } 1\end{array}$ & $491.9 \pm 36.7$ & $568.4 \pm 84.5$ & $772.8 \pm 72.8$ & $654.6 \pm 57.2$ & 3.91 & $0.02^{\mathrm{b}}$ \\
\hline 212015_x_at & PTBP1 & $\begin{array}{l}\text { polypyrimidine tract-binding } \\
\text { protein } 1\end{array}$ & $362.4 \pm 28.4$ & $388.6 \pm 67.6$ & $536.2 \pm 44.8$ & $448.3 \pm 40.2$ & 3.04 & $0.046^{\mathrm{b}}$ \\
\hline 216306_x_at & PTBP1 & $\begin{array}{l}\text { polypyrimidine tract-binding } \\
\text { protein } 1\end{array}$ & $655 \pm 76.3$ & $762.9 \pm 66.6$ & $1,021.3 \pm 100.7$ & $962.4 \pm 90.4$ & 4.26 & $0.01^{\mathrm{b}}$ \\
\hline 212262_at & QKI & homolog of mouse quaking QKI & $534.1 \pm 33.4$ & $673.4 \pm 56.8$ & $758.8 \pm 46.4$ & $820.6 \pm 100.2$ & 4.49 & $0.01^{\mathrm{b}}$ \\
\hline 212636_at & QKI & homolog of mouse quaking QKI & $7,246.6 \pm 762.9$ & $9,010.5 \pm 882.5$ & $11,098.5 \pm 793.3$ & $10,982.3 \pm 1262.1$ & 4.24 & $0.01^{\mathrm{b}}$ \\
\hline 215089_s_at & RBM10 & RNA-binding motif protein 10 & $261.3 \pm 48.8$ & $219.1 \pm 47.1$ & $463.6 \pm 48.3$ & $314.2 \pm 84.7$ & 3.45 & $0.03^{\mathrm{b}}$ \\
\hline 207941_s_at & RBM39 & RNA-binding motif protein 39 & $3,192.2 \pm 118.6$ & $3,276.6 \pm 263$ & $4,108.2 \pm 117.8$ & $3,436.4 \pm 238.7$ & 5.32 & $0.005^{\mathrm{b}}$ \\
\hline 219507_at & RSRC1 & $\begin{array}{l}\text { arginine/serine-rich } \\
\text { coiled-coil } 1\end{array}$ & $66.8 \pm 12.9$ & $85.2 \pm 18.1$ & $123.3 \pm 8.45$ & $159.6 \pm 30.8$ & 5.1 & $0.006^{\mathrm{b}}$ \\
\hline 221768_at & SFPQ & $\begin{array}{l}\text { splicing factor proline/ } \\
\text { glutamine rich }\end{array}$ & $794.7 \pm 40.6$ & $861.6 \pm 83.5$ & $1,137.9 \pm 126.6$ & $780.4 \pm 104.9$ & 3.37 & $0.03^{\mathrm{b}}$ \\
\hline 216364_s_at & AFF2 & AF4/FMR2 family, member 2 & $15.2 \pm 1.96$ & $24.1 \pm 4.26$ & $23.1 \pm 6.56$ & $65.9 \pm 23.5$ & 3.92 & $0.02^{\mathrm{c}}$ \\
\hline 203809_s_at & AKT2 & $\begin{array}{l}\text { RAC-beta serine/threonine- } \\
\text { protein kinase }\end{array}$ & $64.4 \pm 13.9$ & $75.9 \pm 9.3$ & $79.9 \pm 11.6$ & $131 \pm 20$ & 4.16 & $0.02^{\mathrm{c}}$ \\
\hline 202256_at & CD2BP2 & $\begin{array}{l}\text { CD2 (cytoplasmic tail)-binding } \\
\text { protein } 2\end{array}$ & $365 \pm 20.1$ & $381.7 \pm 34.1$ & $411.4 \pm 28.2$ & $522.8 \pm 43.4$ & 4.98 & $0.007^{\mathrm{c}}$ \\
\hline 209056_s_at & CDC5L & cell division cycle 5 -like protein & $676.3 \pm 36.6$ & $623.8 \pm 48.4$ & $578.1 \pm 25.7$ & $492.2 \pm 40.9$ & 4.21 & $0.01^{\mathrm{c}}$ \\
\hline 219539_at & GEMIN6 & $\begin{array}{l}\text { gem (nuclear organelle)- } \\
\text { associated protein } 6\end{array}$ & $143.5 \pm 18.9$ & $130.8 \pm 14.7$ & $174 \pm 15.4$ & $214.5 \pm 26.5$ & 3.5 & $0.03^{\mathrm{c}}$ \\
\hline 210588_x_at & HNRNPH3 & $\begin{array}{l}\text { heterogeneous nuclear } \\
\text { ribonucleoprotein H3 }\end{array}$ & $486.2 \pm 39.8$ & $578.9 \pm 62.8$ & $424.5 \pm 34.7$ & $689.7 \pm 92.5$ & 3.82 & $0.02^{\mathrm{c}}$ \\
\hline 202072_at & HNRNPL & $\begin{array}{l}\text { heterogeneous nuclear } \\
\text { ribonucleoprotein L }\end{array}$ & $692.3 \pm 82.2$ & $746.9 \pm 99$ & $694.9 \pm 63.6$ & $380 \pm 81.3$ & 3.86 & $0.02^{\mathrm{c}}$ \\
\hline 220764_at & LOC100132773 & $\begin{array}{l}\text { serine/threonine-protein } \\
\text { phosphatase 4-regulatory } \\
\text { subunit 2-like }\end{array}$ & $50 \pm 13.9$ & $21.1 \pm 9.33$ & $62.5 \pm 15.4$ & $96.8 \pm 25.3$ & 3.25 & $0.04^{\mathrm{c}}$ \\
\hline 217415_at & POLR2A & $\begin{array}{l}\text { polymerase (RNA) II } \\
\text { (DNA-directed) polypeptide A, } \\
220 \mathrm{kDa}\end{array}$ & $76.1 \pm 13.3$ & $56.8 \pm 14.3$ & $81 \pm 14.2$ & $136 \pm 26.6$ & 3.55 & $0.03^{\mathrm{c}}$ \\
\hline 202634_at & POLR2K & $\begin{array}{l}\text { polymerase (RNA) II } \\
\text { (DNA-directed) polypeptide K, } \\
7.0 \mathrm{kDa}\end{array}$ & $850 \pm 66.5$ & $836.7 \pm 57.5$ & $789.2 \pm 34.9$ & $590.1 \pm 68.8$ & 3.95 & $0.02^{\mathrm{c}}$ \\
\hline 202494_at & PPIE & $\begin{array}{l}\text { peptidylprolyl isomerase E } \\
\text { (cyclophilin E) }\end{array}$ & $206.1 \pm 16.9$ & $281.4 \pm 53.7$ & $262 \pm 18.9$ & $360.4 \pm 40.7$ & 3.7 & $0.02^{\mathrm{c}}$ \\
\hline 203103_s_at & PRPF19 & $\begin{array}{l}\text { PRP19/PSO4 pre-mRNA- } \\
\text { processing factor } 19 \text { homolog }\end{array}$ & $1,384.9 \pm 112.7$ & $1,182.1 \pm 119.7$ & $1,138.3 \pm 89.3$ & $879.1 \pm 86.8$ & 4.01 & $0.02^{\mathrm{c}}$ \\
\hline
\end{tabular}


Table 2 (continued)

\begin{tabular}{|c|c|c|c|c|c|c|c|c|}
\hline Probe & Gene & Description & Control & Incipient & Moderate & Severe & $\begin{array}{l}\text { ANOVA } \\
\text { F }\end{array}$ & $\begin{array}{l}\text { ANOVA } \\
\mathrm{p}\end{array}$ \\
\hline 218088_s_at & RRAGC & Ras-related GTP-binding C & $1,081.5 \pm 52.4$ & $1,013.4 \pm 82.1$ & $1,218.4 \pm 67.1$ & $1,411.2 \pm 69.2$ & 6.43 & $0.002^{\mathrm{c}}$ \\
\hline 201070_x_at & SF3B1 & $\begin{array}{l}\text { splicing factor } 3 \mathrm{~b} \text {, subunit } 1 \text {, } \\
155 \mathrm{kDa}\end{array}$ & $501.4 \pm 44.3$ & $517.2 \pm 28.9$ & $536.4 \pm 53.8$ & $829.1 \pm 163.4$ & 3.27 & $0.04^{\mathrm{c}}$ \\
\hline 217608_at & SFRS12IP1 & SREK1-interacting protein 1 & $68.4 \pm 6.13$ & $62.8 \pm 10.1$ & $86.7 \pm 13.1$ & $122.1 \pm 12.6$ & 6.02 & $0.003^{\mathrm{c}}$ \\
\hline 218493_at & SNRNP25 & $\begin{array}{l}\text { small nuclear } \\
\text { ribonucleoprotein, } 25 \mathrm{kDa} \\
\text { (U11/U12) }\end{array}$ & $1,080.4 \pm 83$ & $1,070.7 \pm 113.3$ & $1,019.8 \pm 59.2$ & $714.5 \pm 41.7$ & 4.52 & $0.01^{\mathrm{c}}$ \\
\hline 200826_at & SNRPD2 & $\begin{array}{l}\text { small nuclear ribonucleoprotein } \\
\text { D2 polypeptide, } 16.5 \mathrm{kDa}\end{array}$ & $2,611.4 \pm 94$ & $2,699 \pm 193.7$ & $2,648.4 \pm 106.6$ & $2,012.6 \pm 251.4$ & 3.64 & $0.03^{\mathrm{c}}$ \\
\hline 211439_at & SRSF7 & $\begin{array}{l}\text { serine/arginine-rich splicing } \\
\text { factor } 7 \text { (9G8) }\end{array}$ & $137.5 \pm 19.8$ & $199.5 \pm 43.3$ & $124.1 \pm 11.9$ & $235.7 \pm 31.4$ & 3.61 & $0.03^{\mathrm{c}}$ \\
\hline 210180_s_at & TRA2B & $\begin{array}{l}\text { transformer-2 protein homolog } \\
\text { beta }\end{array}$ & $22.2 \pm 4.97$ & $6.97 \pm 1.06$ & $16.9 \pm 6.27$ & $34.6 \pm 9.26$ & 3.3 & $0.04^{\mathrm{c}}$ \\
\hline 206067_s_at & WT1 & Wilms tumor 1 & $8.11 \pm 1.46$ & $12.1 \pm 3.63$ & $13.3 \pm 3.26$ & $21.1 \pm 3.38$ & 3.41 & $0.03^{\mathrm{c}}$ \\
\hline 214759_at & WTAP & $\begin{array}{l}\text { Wilms tumor 1-associated } \\
\text { protein }\end{array}$ & $142.9 \pm 25.7$ & $174.5 \pm 19.6$ & $130 \pm 12$ & $216.4 \pm 26$ & 2.99 & $0.049^{\mathrm{c}}$ \\
\hline
\end{tabular}

${ }^{\mathrm{a}}$ Incipient $\mathrm{p}<0.05$ (Fisher LSD); ${ }^{\mathrm{b}}$ moderate $\mathrm{p}<0.05$ (Fisher LSD); ${ }^{\mathrm{c}}$ severe $\mathrm{p}<0.05$ (Fisher LSD).

products are known to be involved (either directly or indirectly) in RNA splicing were screened.

\section{GDS810 Microarray}

The GDS810 microarray published by Blalock et al. [23] assessed gene expression changes in the hippocampal CA1 subfield from a total of 31 subjects -9 controls and 22 AD subjects of varying AD severity. A total of 22,286 genes were tested and of these, 499 were involved in RNA splicing. Of the 499 genes, only 45 were found to be altered from the incipient stage of AD to the severe stage (table 2). Specifically, 6 of the 45 genes identified were significantly changed in subjects with incipient AD. Interestingly, hnrnpA3 was the only gene that showed a significant decrease in expression, whereas NUDT21, LSM5, hnrnpUL1, RBM8A, and RBMY1J were significantly increased in expression. In subjects with moderate AD, the expression of 18 genes was found to be significantly altered compared to controls. Of these, 5 genes were significantly decreased, whereas 13 genes were significantly increased. In subjects with severe AD, 21 genes were found to be significantly changed compared to controls. Of these, 6 genes showed a significantly reduced expression, whereas 15 showed a significantly increased expression.

It is worth noting that while 45 genes were found to be significantly altered during AD progression, the expression of 34 genes trended towards statistical significance (one-way ANOVA: $0.05<\mathrm{p}<0.1$ ) (table 3). Of these, 11 were decreased in expression and 23 were increased in expression.

\section{GSE28146 Microarray}

In the GSE28146 microarray published by Blalock et al. [24], grey matter of the hippocampal CA1 subfield was selectively isolated using laser capture microdissection from the same subjects and analyzed for gene expression changes. Using this dataset, the expression of splice-regulatory proteins that were significantly altered or showed a trend towards statistical significance in AD in the GDS810 microarray was screened to determine whether changes in gene expression were specific to the grey matter. Of the 45 genes that were significantly changed throughout the course of AD in the GDS810 microarray study, only QKI, PTBP1, and SFPQ were significantly altered in the GSE28146 microarray (table 4). Of the 34 genes that 
Wong: Altered Expression of RNA Splicing Proteins in Alzheimer's Disease Patients:

Evidence from Two Microarray Studies

Table 3. Microarray gene expression changes in the CA1 hippocampus $(0.05<\mathrm{p}<0.1$; GDS810 microarray $)$

\begin{tabular}{|c|c|c|c|c|c|c|c|c|}
\hline Probe & Gene & Description & Control & Incipient & Moderate & Severe & $\begin{array}{l}\text { ANOVA } \\
\text { F }\end{array}$ & $\begin{array}{l}\text { ANOVA } \\
\mathrm{p}\end{array}$ \\
\hline 200041_s_at & BAT1 & $\begin{array}{l}\text { spliceosome RNA helicase } \\
\text { DDX39B }\end{array}$ & $2,839.2 \pm 220.5$ & $3,233.4 \pm 249$ & $3,948.7 \pm 274$ & $3,076.4 \pm 412.2$ & 2.9 & $0.05^{\mathrm{a}}$ \\
\hline 209055_s_at & CDC5L & cell division cycle 5-like protein & $130.2 \pm 19.2$ & $256.1 \pm 66.6$ & $126.1 \pm 21.6$ & $149.1 \pm 35.9$ & 2.53 & $0.08^{\mathrm{a}}$ \\
\hline 215045_at & CELF3 & $\begin{array}{l}\text { CUGBP, Elav-like family } \\
\text { member } 3\end{array}$ & $631.6 \pm 98.2$ & $537 \pm 59.6$ & $669.9 \pm 87$ & $389.9 \pm 43.4$ & 2.33 & $0.1^{\mathrm{a}}$ \\
\hline 203947_at & CSTF3 & $\begin{array}{l}\text { cleavage stimulation factor, } 3^{\prime} \\
\text { pre-RNA, subunit } 3,77 \mathrm{kDa}\end{array}$ & $802 \pm 58.7$ & $786.8 \pm 65.6$ & $740.2 \pm 68.9$ & $1,000 \pm 98.5$ & 2.34 & $0.1^{\mathrm{a}}$ \\
\hline 219149_x_at & DBR1 & debranching enzyme homolog 1 & $129.2 \pm 11.6$ & $173.5 \pm 15.8$ & $171 \pm 9.40$ & $172.3 \pm 18.2$ & 2.73 & $0.06^{\mathrm{a}}$ \\
\hline 219121_s_at & ESRP1 & $\begin{array}{l}\text { epithelial splicing-regulatory } \\
\text { protein } 1\end{array}$ & $73.9 \pm 19.5$ & $40.1 \pm 7.51$ & $31.7 \pm 3.72$ & $81.4 \pm 21.8$ & 2.47 & $0.08^{\mathrm{a}}$ \\
\hline 200959_at & FUS & fused in sarcoma & $797.9 \pm 56.5$ & $999.8 \pm 59.8$ & $821.5 \pm 32.5$ & $841.9 \pm 73.8$ & 2.5 & $0.08^{\mathrm{a}}$ \\
\hline 215744_at & FUS & fused in sarcoma & $127.3 \pm 14.9$ & $88.3 \pm 21.6$ & $122.8 \pm 11.8$ & $160.7 \pm 26.1$ & 2.3 & $0.1^{\mathrm{a}}$ \\
\hline 202354_s_at & GTF2F1 & $\begin{array}{l}\text { general transcription factor IIF, } \\
\text { polypeptide } 1,74 \mathrm{kDa}\end{array}$ & $95.5 \pm 15.5$ & $144.4 \pm 20$ & $75.2 \pm 21.9$ & $80 \pm 19.5$ & 2.53 & $0.08^{\mathrm{a}}$ \\
\hline 35201_at & HNRNPL & $\begin{array}{l}\text { heterogeneous nuclear } \\
\text { ribonucleoprotein L }\end{array}$ & $1,963.2 \pm 158$ & $2,212 \pm 171$ & $2,120.6 \pm 118.2$ & $1,689.6 \pm 84.3$ & 2.45 & $0.09^{\mathrm{a}}$ \\
\hline 214918_at & HNRNPM & $\begin{array}{l}\text { heterogeneous nuclear } \\
\text { ribonucleoprotein M }\end{array}$ & $66.1 \pm 10.2$ & $72.8 \pm 16.7$ & $90.9 \pm 21.3$ & $136.3 \pm 27$ & 2.66 & $0.07^{\mathrm{a}}$ \\
\hline 219814_at & MBNL3 & muscleblind-like 3 & $33.5 \pm 9.70$ & $13.5 \pm 3.45$ & $42 \pm 16$ & $67.5 \pm 20.9$ & 2.39 & $0.09^{a}$ \\
\hline 212718_at & PAPOLA & poly(A) polymerase alpha & $2,442.8 \pm 240.9$ & $2,252.2 \pm 428.9$ & $2,644.5 \pm 251.4$ & $3,686 \pm 610.5$ & 2.55 & $0.08^{\mathrm{a}}$ \\
\hline 203378_at & PCF11 & $\begin{array}{l}\text { cleavage and polyadenylation } \\
\text { factor subunit, homolog }\end{array}$ & $491 \pm 41.8$ & $533.8 \pm 55.3$ & $673.7 \pm 50.7$ & $547.9 \pm 46.1$ & 2.81 & $0.06^{\mathrm{a}}$ \\
\hline 210183_x_at & PNN & $\begin{array}{l}\text { pinin, desmosome-associated } \\
\text { protein }\end{array}$ & $4,076.8 \pm 556.7$ & $3,036.2 \pm 149.6$ & $4,261.8 \pm 184.9$ & $3,148 \pm 358.9$ & 2.65 & $0.07^{\mathrm{a}}$ \\
\hline 214144_at & POLR2D & $\begin{array}{l}\text { polymerase (RNA) II (DNA } \\
\text { directed) polypeptide D }\end{array}$ & $161.8 \pm 14.8$ & $131.9 \pm 7.06$ & $136.3 \pm 13.4$ & $182.6 \pm 19.5$ & 2.5 & $0.08^{\mathrm{a}}$ \\
\hline 221649_s_at & PPAN & peter pan homolog & $173.8 \pm 10.5$ & $152.8 \pm 13.8$ & $169.7 \pm 18$ & $249.6 \pm 50.5$ & 2.51 & $0.08^{\mathrm{a}}$ \\
\hline 220553_s_at & PRPF39 & $\begin{array}{l}\text { PRP39 pre-mRNA processing } \\
\text { factor } 39 \text { homolog }\end{array}$ & $530 \pm 46.5$ & $481.7 \pm 40.3$ & $535.7 \pm 45.5$ & $381.3 \pm 47.9$ & 2.35 & $0.09^{\mathrm{a}}$ \\
\hline 202126_at & PRPF4B & $\begin{array}{l}\text { PRP4 pre-mRNA processing } \\
\text { factor } 4 \text { homolog B }\end{array}$ & $1,002.1 \pm 52.7$ & $1,075.9 \pm 40.8$ & $1,159.1 \pm 62.6$ & $1,198.6 \pm 71.7$ & 2.37 & $0.09^{\mathrm{a}}$ \\
\hline 217857_s_at & RBM8A & RNA-binding motif protein 8A & $22.1 \pm 4.44$ & $42.5 \pm 10.8$ & $34.6 \pm 11.8$ & $11.5 \pm 1.01$ & 2.56 & $0.08^{\mathrm{a}}$ \\
\hline 208307_at & RBMY1J & $\begin{array}{l}\text { RNA-binding motif protein, } \\
\text { Y-linked, family 1, member J }\end{array}$ & $26.5 \pm 5.12$ & $33 \pm 14.2$ & $39.3 \pm 9.54$ & $78.7 \pm 26.8$ & 2.41 & $0.09^{\mathrm{a}}$ \\
\hline 209381_x_at & SF3A2 & $\begin{array}{l}\text { splicing factor } 3 \mathrm{a} \text {, subunit } \\
2,66 \mathrm{kDa}\end{array}$ & $172.1 \pm 41.3$ & $223.2 \pm 58.3$ & $368.3 \pm 62.7$ & $515.8 \pm 179.2$ & 2.69 & $0.07^{\mathrm{a}}$ \\
\hline 221263_s_at & SF3B5 & $\begin{array}{l}\text { splicing factor } 3 \mathrm{~b}, \text { subunit } \\
5,10 \mathrm{kDa}\end{array}$ & $1,134.9 \pm 70.8$ & $955.7 \pm 73.6$ & $914.9 \pm 29$ & $1,011.1 \pm 72.1$ & 2.46 & $0.08^{\mathrm{a}}$ \\
\hline 213505_s_at & SFRS14 & $\begin{array}{l}\text { putative splicing factor, arginine/ } \\
\text { serine-rich } 14\end{array}$ & $832.2 \pm 47.1$ & $779.5 \pm 51$ & $749.8 \pm 67.8$ & $630.4 \pm 38.6$ & 2.55 & $0.08^{\mathrm{a}}$ \\
\hline 204978_at & SFRS16 & $\begin{array}{l}\text { splicing factor, arginine/ } \\
\text { serine-rich } 16\end{array}$ & $949.1 \pm 114.7$ & $1,131.2 \pm 161.5$ & $1,134.6 \pm 118.2$ & $1,803.3 \pm 405.1$ & 2.92 & $0.05^{\mathrm{a}}$ \\
\hline 212438_at & SNRNP27 & $\begin{array}{l}\text { small nuclear ribonucleoprotein, } \\
27 \mathrm{kDa} \text { (U4/U6.U5) }\end{array}$ & $690.8 \pm 102.8$ & $451.6 \pm 21.4$ & $410.5 \pm 31.7$ & $586.9 \pm 111.1$ & 2.72 & $0.06^{\mathrm{a}}$ \\
\hline 215722_s_at & SNRPA1 & $\begin{array}{l}\text { small nuclear ribonucleoprotein } \\
\text { polypeptide } A^{\prime}\end{array}$ & $232 \pm 11.7$ & $296.7 \pm 33.1$ & $236.5 \pm 19.6$ & $205.4 \pm 31.1$ & 2.4 & $0.09^{\mathrm{a}}$ \\
\hline 208821_at & SNRPB & $\begin{array}{l}\text { small nuclear ribonucleoprotein } \\
\text { polypeptides B and B1 }\end{array}$ & $337.4 \pm 28.8$ & $326.6 \pm 15.5$ & $284 \pm 26.3$ & $415.6 \pm 59.5$ & 2.33 & $0.1^{\mathrm{a}}$ \\
\hline 208610_s_at & SRRM2 & $\begin{array}{l}\text { serine/arginine repetitive } \\
\text { matrix } 2\end{array}$ & $922.4 \pm 118.4$ & $1,019.6 \pm 110.7$ & $1,177.7 \pm 148.3$ & $1,986.8 \pm 611$ & 2.53 & $0.08^{\mathrm{a}}$ \\
\hline 206989_s_at & SRSF2IP & $\begin{array}{l}\text { SR-related CTD-associated } \\
\text { factor } 11\end{array}$ & $449.9 \pm 27.2$ & $563.6 \pm 42.9$ & $572 \pm 41.3$ & $486.7 \pm 35.9$ & 2.77 & $0.06^{\mathrm{a}}$ \\
\hline 201129_at & SRSF7 & $\begin{array}{l}\text { serine/arginine-rich splicing } \\
\text { factor } 7 \text { (9G8) }\end{array}$ & $1,286.8 \pm 76.1$ & $1,001 \pm 79.4$ & $1,048 \pm 100.7$ & $1,008.9 \pm 109.5$ & 2.37 & $0.09^{a}$ \\
\hline 202553_s_at & SYF2 & $\begin{array}{l}\text { SYF2 homolog, RNA splicing } \\
\text { factor }\end{array}$ & $998 \pm 90$ & $1,163.6 \pm 71.5$ & $1,307.8 \pm 75.3$ & $1,189.4 \pm 67.7$ & 2.87 & $0.06^{\mathrm{a}}$ \\
\hline 200020_at & TARDBP & $\begin{array}{l}\text { TAR DNA-binding protein } \\
\text { (TDP-43) }\end{array}$ & $1,269.4 \pm 60.2$ & $1,455.1 \pm 135.5$ & $1,201.1 \pm 53.4$ & $1,592.9 \pm 176.9$ & 2.58 & $0.08^{\mathrm{a}}$ \\
\hline 214814_at & YTHDC1 & YTH domain containing 1 & $72.1 \pm 13.8$ & $118.9 \pm 14.2$ & $94 \pm 14.4$ & $131.5 \pm 22.6$ & 2.7 & $0.07^{\mathrm{a}}$ \\
\hline 202126_at & PRPF4B & $\begin{array}{l}\text { PRP4 pre-mRNA processing } \\
\text { factor } 4 \text { homolog B }\end{array}$ & $2,050.7 \pm 211.2$ & $1,330.4 \pm 124.9$ & $2,149.5 \pm 267.1$ & $2,313 \pm 353.5$ & 2.8 & $0.06^{\mathrm{b}}$ \\
\hline 200020_at & TARDBP & $\begin{array}{l}\text { TAR DNA-binding protein } \\
\text { (TDP-43) }\end{array}$ & $4,252.9 \pm 372.4$ & $4,204.2 \pm 262.5$ & $3,107.2 \pm 229.7$ & $4,109.3 \pm 488$ & 2.56 & $0.08^{\mathrm{b}}$ \\
\hline
\end{tabular}

${ }^{a}$ GDS810 microarray. ${ }^{\text {b }}$ GSE28146 microarray. 
Table 4. Microarray gene expression changes in the CA1 hippocampus (grey matter laser capture microdissection; GSE28146 microarray)

\begin{tabular}{llcccccc}
\hline Probe & Gene & Control & Incipient & Moderate & Severe & $\begin{array}{l}\text { ANOVA } \\
\text { F }\end{array}$ & $\begin{array}{l}\text { ANOVA } \\
\text { p }\end{array}$ \\
\hline 212262_at & QKI & $534.1 \pm 33.4$ & $673.4 \pm 56.8$ & $758.8 \pm 46.4$ & $820.6 \pm 100.2$ & 3.45 & $0.03^{\mathrm{a}}$ \\
211271_x_at & PTBP1 & $491.9 \pm 36.7$ & $568.4 \pm 84.5$ & $772.8 \pm 72.8$ & $654.6 \pm 57.2$ & 3.48 & $0.03^{\mathrm{b}}$ \\
221768_at & SFPQ & $794.7 \pm 40.6$ & $861.6 \pm 83.5$ & $1,137.9 \pm 126.6$ & $780.4 \pm 104.9$ & 3.95 & $0.02^{\mathrm{b}}$ \\
201129_at & SRSF7 & $1,286.8 \pm 76.1$ & $1,001 \pm 79.4$ & $1,048 \pm 100.7$ & $1,008.9 \pm 109.5$ & 3.79 & $0.02^{\mathrm{b}}$ \\
204978_at & SFRS16 & $949.1 \pm 114.7$ & $1,131.2 \pm 161.5$ & $1,134.6 \pm 118.2$ & $1,803.3 \pm 405.1$ & 3.5 & $0.03^{\mathrm{b}}$ \\
214814_at & YTHDC1 & $72.1 \pm 13.8$ & $118.9 \pm 14.2$ & $94 \pm 14.4$ & $131.5 \pm 22.6$ & 5.59 & $0.004^{\mathrm{c}}$ \\
\hline
\end{tabular}

${ }^{\mathrm{a}}$ Incipient $\mathrm{p}<0.05$ (Fisher LSD); ${ }^{\mathrm{b}}$ moderate $\mathrm{p}<0.05$ (Fisher LSD); ${ }^{\mathrm{c}}$ severe $\mathrm{p}<0.05$ (Fisher LSD).

Table 5. Correlations: splice-regulatory protein gene expression

\begin{tabular}{lll}
\hline & $\mathrm{r}$ & $\mathrm{p}$ \\
\hline PTBP1 (GSE28146) & & \\
PTBP1 (GDS810) & 0.36 & 0.05 \\
\hline QKI (GSE28146) & 0.48 & $0.01^{\mathrm{a}}$ \\
QKI (GDS810) & \\
\hline $\begin{array}{l}\text { SFPQ (GSE28146) } \\
\text { SFPQ (GDS810) }\end{array}$ & 0.18 & 0.35 \\
\hline $\begin{array}{l}\text { SRSF7 (GSE28146) } \\
\text { SRSF7 (GDS810) }\end{array}$ & 0.50 & $0.01^{\mathrm{a}}$ \\
\hline $\begin{array}{l}\text { SFRS16 (GSE28146) } \\
\text { SFRS16 (GDS810) }\end{array}$ & -0.15 & 0.42 \\
\hline YTHDC1 (GSE28146) & & \\
YTHDC1 (GDS810) & 0.11 & \\
\hline \multicolumn{1}{c}{ a $p<0.05}$. & & \\
\hline
\end{tabular}

trended towards significance in the GDS810 microarray study, SRSF7, SFRS16, and YTHDC1 were found to be significantly changed in the GSE28146 study.

It was next determined whether the splice-regulatory proteins found to be significantly altered in both the GDS810 and GSE28146 microarray studies correlated in their gene expression pattern over the disease duration. Interestingly, QKI and SRSF7 were the only two genes showing concordance (table 5).

\section{Gene Expression Correlations between Splice-Regulatory Proteins and AD Susceptibility Genes}

Next, it was determined whether changes in splice-regulatory protein expression found to be significant in both the GDS810 and GSE28146 microarray studies correlated with gene expression changes of various transcripts reported to be altered in AD. These included progranulin (GRN), microtubule-associated protein tau (MAPT), presenilin-1 (PSEN1), presenilin-2 (PSEN2), presenilin enhancer protein 2 (PSENEN), amyloid beta (A4) precursor protein (APP), apolipoprotein E (APOE), TrkB (NTRK2), and brain-derived neurotrophic factor (BDNF) [25-29]. While the expression of most transcripts correlated with expression levels of the splice-regulatory proteins in their respective studies, only APP and TrkB tran- 
Wong: Altered Expression of RNA Splicing Proteins in Alzheimer's Disease Patients: Evidence from Two Microarray Studies

Table 6. Correlations: splice-regulatory proteins and AD susceptibility gene transcripts (GDS810)

\begin{tabular}{|c|c|c|}
\hline & $\mathrm{r}$ & $\mathrm{p}$ \\
\hline PSEN1, QKI & 0.47 & 0.01 \\
\hline MAPT, SFRS16 & -0.44 & 0.01 \\
\hline PSEN2, QKI & 0.53 & 0.002 \\
\hline PSEN2, SRSF7 & -0.42 & 0.02 \\
\hline PSEN2, SRSF7 & -0.45 & 0.01 \\
\hline BDNF, SFPQ & -0.41 & 0.03 \\
\hline NTRK2, SFRS16 & 0.61 & $0.0003^{\mathrm{a}}$ \\
\hline NTRK2, YTHDC1 & 0.55 & 0.002 \\
\hline PSEN1, SFRS16 & -0.39 & 0.03 \\
\hline APP, QKI & 0.49 & 0.01 \\
\hline APP, SRSF7 & -0.46 & 0.01 \\
\hline APP, SFRS16 & 0.87 & 0.0000000003 \\
\hline APP, YTHDC1 & 0.64 & $0.0001^{\mathrm{a}}$ \\
\hline GRN, PTBP1 & 0.39 & 0.03 \\
\hline NTRK2, SFRS16 & -0.65 & $0.0001^{\mathrm{a}}$ \\
\hline APP, SFRS16 & -0.54 & 0.002 \\
\hline APP, YTHDC1 & -0.38 & $0.04^{\mathrm{a}}$ \\
\hline GRN, QKI & 0.40 & 0.03 \\
\hline PSENEN, SFRS16 & -0.55 & 0.002 \\
\hline NTRK2, SRSF7 & -0.43 & $0.02^{\mathrm{a}}$ \\
\hline NTRK2, SFPQ & 0.36 & $0.05^{\mathrm{a}}$ \\
\hline NTRK2, SFRS16 & -0.44 & $0.01^{\mathrm{a}}$ \\
\hline
\end{tabular}

a $\mathrm{p}<0.05$ in GDS810 and GSE28146 microarrays.

scripts correlated significantly with the same splice-regulatory proteins in the two studies (tables 6, 7). Two APP transcripts showed significant positive correlations with YTHDC1, whereas one transcript showed a negative correlation. The TrkB transcripts showing significant correlations with splice-regulatory protein expression were those encoding the C-terminal truncated TrkB isoform TrkB-TK-. TrkB-TK- transcripts correlated negatively with SRSF7 and SFRS16 but positively with SFPQ expression throughout AD progression.

\section{Discussion}

In the two microarray datasets utilized in this study, most changes in gene expression were found to occur during the moderate to severe stages of $\mathrm{AD}$, periods coinciding with gross pathological brain changes and cognitive decline [30], whereas fewer changes occurred during the incipient stage of AD. Interestingly, of the 45 splice-regulatory proteins whose gene expression was found to be significantly altered in the GDS810 microarray study, only 
Table 7. Correlations: splice-regulatory proteins and AD susceptibility gene transcripts (GSE28146)

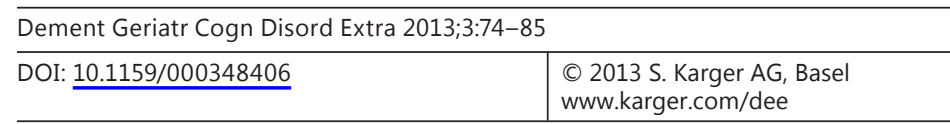

Wong: Altered Expression of RNA Splicing Proteins in Alzheimer's Disease Patients: Evidence from Two Microarray Studies

\begin{tabular}{lll}
\hline & $\mathrm{r}$ & $\mathrm{p}$ \\
\hline GRN, SFPQ & -0.45 & 0.01 \\
\hline APOE, PTBP1 & 0.37 & 0.05 \\
\hline PSEN1, SFPQ & 0.48 & 0.01 \\
\hline MAPT, SFPQ & -0.63 & 0.0002 \\
\hline NTRK2, QKI & -0.47 & 0.01 \\
\hline PSEN1, SFPQ & 0.37 & 0.05 \\
\hline APP, YTHDC1 & 0.49 & $0.01^{\mathrm{a}}$ \\
\hline GRN, YTHDC1 & 0.44 & 0.01 \\
\hline NTRK2, SRSF7 & -0.44 & $0.02^{\mathrm{a}}$ \\
\hline NTRK2, QKI & 0.44 & 0.01 \\
\hline NTRK2, SFRS16 & -0.41 & $0.03^{\mathrm{a}}$ \\
\hline NTRK2, SFPQ & 0.39 & $0.03^{\mathrm{a}}$ \\
\hline NTRK2, SRSF7 & -0.52 & $0.003^{\mathrm{a}}$ \\
\hline
\end{tabular}

${ }^{\mathrm{a}} \mathrm{p}<0.05$ in GDS810 and GSE28146 microarrays.

6 were significant in the GSE28146 study; moreover, these significant changes in gene expression occurred during the incipient stages of AD. This finding suggests that changes in splice-regulatory protein expression may occur in the grey matter early in the disease process, prior to gross pathological brain changes. Indeed, when the GDS810 and GSE28146 microarrays were assessed to determine whether those splicing proteins found to be significantly altered in both microarray studies correlated in their gene expression pattern over the disease duration, QKI and SRSF7 were the only two genes showing concordance. The lack of concordance in splice-regulatory protein expression between the two microarray studies suggests that the majority of gene expression changes in the GDS810 study were likely influenced by changes in the white matter, which was largely excluded by the laser capture microdissection in the GSE28146 study.

\section{Relationship between Splice-Regulatory Proteins and AD Susceptibility Genes}

When it was assessed whether the expression of splice-regulatory proteins found to be significantly altered in AD correlated with the expression levels of AD susceptibility genes, significant correlations in APP and TrkB-TK- transcripts throughout AD progression in both the GDS810 and GSE28146 microarray studies suggest that changes in gene expression may be primarily influenced by changes in the grey matter, which is consistent with the cellular expression of APP (expressed primarily in neurons and glia and to a lesser extent in endothelial cells) and TrkB-TK- (expressed principally by astrocytes and to a lesser extent by neurons) [31-33]. The APP transcripts showing a significant correlation with YTHDC1 were targeted by pan probes, whereas the TrkB transcripts showing significant correlations with SRSF7, SFRS16, and SPQF were targeted by probes specific for the transcript variant encoding TrkB-TK-. These findings suggest that YTHDC1 may be involved in regulating constitutive splicing of the APP pre-mRNA rather than regulating alternative splicing of specific exons. 
The TrkB-TK- transcript variant encodes a TrkB protein isoform truncated at the C terminus and in comparison to the full-length receptor, and thus cannot partake in classical receptor tyrosine kinase signaling [34-36]. This is due to alternative exon splicing of the pre-mRNA where exon 16 is incorporated into the final transcript. Exon 16 encodes a translation stop codon and polyadenylation sequence; thus, transcripts are differentially regulated posttranscriptionally $[34,36]$. The finding that TrkB-TK- transcript levels correlated with the same splice-regulatory proteins significantly altered in both microarray studies implicates these splice-regulatory proteins as potential regulators of TrkB-TK- expression. In particular, SRSF7 has previously been demonstrated to be a regulator of tau alternative splicing [20]. Abnormal ratios of tau isoforms $3 \mathrm{R}$ and $4 \mathrm{R}$ (imperfect repeats of approximately 32 amino acids in the microtubule-binding domain) lead to tau aggregation and neurofibrillary tangle formation. The generation of tau isoforms $3 \mathrm{R}$ and $4 \mathrm{R}$ is determined by alternative mRNA splicing of exon 10 [20-22], and point mutations in the splice-regulatory region affecting SRSF7 binding have been shown to modulate inclusion/exclusion of tau exon 10 [20]. In both the GDS810 and GSE28146 microarray studies, the probes targeted to MAPT transcripts (gene encoding tau) were not specific for exon 10 detection, thus no significant correlation between changes in SRSF7 and MAPT expression was found. However, MAPT expression is likely to be regulated by additional mechanisms. Considering that SRSF7 was one of only two splice-regulatory proteins to be significantly and positively correlated between the two microarray studies and is significantly correlated with TrkB-TK- transcript levels in the two studies, the findings reported here implicate SRSF7 as a potential regulator of TrkB pre-mRNA splicing in the production of TrkB-TK- alternative transcripts in the grey matter. The lack of concordance between SFRS16 and SPQF gene expression changes between the GDS810 and GSE28146 microarray studies suggests that these two splice-regulatory proteins may function in regulating TrkB-TK- transcript levels in the white matter.

\section{Conclusion}

Dysregulation in splice-regulatory protein gene expression can adversely affect alternative splicing and gene expression of a number of cellular processes. The findings reported in this study offer mechanistic insight into how aberrant changes in alternative transcript expression may occur in $\mathrm{AD}$ and highlight a susceptibility network - splice-regulatory proteins - which can potentially link a number of susceptibility genes/pathways.

\section{Acknowledgements}

J.W. is supported by the Illawarra Health and Medical Research Institute (University of Wollongong), the Centre for Medical Bioscience (University of Wollongong), an Alzheimer's Australia Dementia Research Foundation (AADRF) Grant, and a National Health and Medical Research Council of Australia (NHMRC) Postdoctoral Training Fellowship (568884).

\section{Disclosure Statement}

There are no conflicts of interest and financial disclosures. 
Wong: Altered Expression of RNA Splicing Proteins in Alzheimer's Disease Patients: Evidence from Two Microarray Studies

\section{References}

1 Fratiglioni L, De Ronchi D, Aguero-Torres H: Worldwide prevalence and incidence of dementia. Drugs Aging 1999;15:365-375.

- 2 Ferri CP, Prince M, Brayne C, Brodaty H, Fratiglioni L, Ganguli M, Hall K, Hasegawa K, Hendrie H, Huang Y, Jorm A, Mathers C, Menezes PR, Rimmer E, Scazufca M: Global prevalence of dementia: a Delphi consensus study. Lancet 2005;366:2112-2117.

3 Serrano-Pozo A, Frosch MP, Masliah E, Hyman BT: Neuropathological alterations in Alzheimer disease. Cold Spring Harb Perspect Med 2011;1:a006189.

- 4 Xu Y, Yan J, Zhou P, Li J, Gao H, Xia Y, Wang Q: Neurotransmitter receptors and cognitive dysfunction in Alzheimer's disease and Parkinson's disease. Prog Neurobiol 2012;97:1-13.

5 Rocha de Paula M, Gomez Ravetti M, Berretta R, Moscato P: Differences in abundances of cell-signalling proteins in blood reveal novel biomarkers for early detection of clinical Alzheimer's disease. PLoS One 2011; 6:e17481.

- 6 Lu KP: Pinning down cell signaling, cancer and Alzheimer's disease. Trends Biochem Sci 2004;29:200-209.

- 7 Mattson MP, Chan SL: Neuronal and glial calcium signaling in Alzheimer's disease. Cell Calcium 2003;34:385397.

- 8 Weninger SC, Yankner BA: Inflammation and Alzheimer disease: the good, the bad, and the ugly. Nat Med 2001;7:527-528.

9 Wyss-Coray T: Inflammation in Alzheimer disease: driving force, bystander or beneficial response? Nat Med 2006;12:1005-1015.

10 Shankar GM, Walsh DM: Alzheimer's disease: synaptic dysfunction and Abeta. Mol Neurodegener 2009;4:48.

11 Palop JJ, Mucke L: Amyloid-beta-induced neuronal dysfunction in Alzheimer's disease: from synapses toward neural networks. Nat Neurosci 2010;13:812-818.

12 Palop JJ, Mucke L: Synaptic depression and aberrant excitatory network activity in Alzheimer's disease: two faces of the same coin? Neuromolecular Med 2010;12:48-55.

13 Yanagisawa K: Cholesterol and pathological processes in Alzheimer's disease. J Neurosci Res 2002;70:361366.

14 Shobab LA, Hsiung GY, Feldman HH: Cholesterol in Alzheimer's disease. Lancet Neurol 2005;4:841-852.

15 Graveley BR: Alternative splicing: increasing diversity in the proteomic world. Trends Genet 2001;17:100107.

16 Stamm S, Ben-Ari S, Rafalska I, Tang Y, Zhang Z, Toiber D, Thanaraj TA, Soreq H: Function of alternative splicing. Gene 2005;344:1-20.

-17 Johnson JM, Castle J, Garrett-Engele P, Kan Z, Loerch PM, Armour CD, Santos R, Schadt EE, Stoughton R, Shoemaker DD: Genome-wide survey of human alternative pre-mRNA splicing with exon junction microarrays. Science 2003;302:2141-2144.

18 Faustino NA, Cooper TA: Pre-mRNA splicing and human disease. Genes Dev 2003;17:419-437.

19 Wong J, Garner B, Halliday GM, Kwok JB: Srp20 regulates TrkB pre-mRNA splicing to generate TrkB-Shc transcripts with implications for Alzheimer's disease. J Neurochem 2012;123:159-171.

20 Gao L, Wang J, Wang Y, Andreadis A: Sr protein 9g8 modulates splicing of tau exon 10 via its proximal downstream intron, a clustering region for frontotemporal dementia mutations. Mol Cell Neurosci 2007;34:48-58.

21 Kalbfuss B, Mabon SA, Misteli T: Correction of alternative splicing of tau in frontotemporal dementia and parkinsonism linked to chromosome 17. J Biol Chem 2001;276:42986-42993.

22 Jiang Z, Cote J, Kwon JM, Goate AM, Wu JY: Aberrant splicing of tau pre-mRNA caused by intronic mutations associated with the inherited dementia frontotemporal dementia with parkinsonism linked to chromosome 17. Mol Cell Biol 2000;20:4036-4048.

23 Blalock EM, Geddes JW, Chen KC, Porter NM, Markesbery WR, Landfield PW: Incipient Alzheimer's disease: microarray correlation analyses reveal major transcriptional and tumor suppressor responses. Proc Natl Acad Sci USA 2004;101:2173-2178.

24 Blalock EM, Buechel HM, Popovic J, Geddes JW, Landfield PW: Microarray analyses of laser-captured hippocampus reveal distinct gray and white matter signatures associated with incipient Alzheimer's disease. J Chem Neuroanat 2011;42:118-126.

25 Mills JD, Janitz M: Alternative splicing of mRNA in the molecular pathology of neurodegenerative diseases. Neurobiol Aging 2012;33:1012.e1011-e1024.

-26 Wong J, Higgins MJ, Halliday G, Garner B: Amyloid beta selectively modulates neuronal TrkB alternative transcript expression with implications for Alzheimer's disease. Neuroscience 2012;210:363-374.

27 Ferrer I, Marin C, Rey MJ, Ribalta T, Goutan E, Blanco R, Tolosa E, Marti E: BDNF and full-length and truncated TrkB expression in Alzheimer disease. Implications in therapeutic strategies. J Neuropathol Exp Neurol 1999; 58:729-739.

28 Connor B, Young D, Lawlor P, Gai W, Waldvogel H, Faull RL, Dragunow M: Trk receptor alterations in Alzheimer's disease. Brain Res Mol Brain Res 1996;42:1-17.

29 Albani D, Batelli S, Pesaresi M, Prato F, Polito L, Forloni G, Pantieri R: A novel PSENEN mutation in a patient with complaints of memory loss and a family history of dementia. Alzheimers Dement 2007;3:235-238.

30 Nelson PT, Braak H, Markesbery WR: Neuropathology and cognitive impairment in Alzheimer disease: a complex but coherent relationship. J Neuropathol Exp Neurol 2009;68:1-14. 
-31 Ohira K, Shimizu K, Yamashita A, Hayashi M: Differential expression of the truncated TrkB receptor, T1, in the primary motor and prefrontal cortices of the adult macaque monkey. Neurosci Lett 2005;385:105-109.

32 Ohira K, Hayashi M: Expression of TrkB subtypes in the adult monkey cerebellar cortex. J Chem Neuroanat 2003;25:175-183.

-33 Frisen J, Verge VM, Fried K, Risling M, Persson H, Trotter J, Hokfelt T, Lindholm D: Characterization of glial TrkB receptors: differential response to injury in the central and peripheral nervous systems. Proc Natl Acad Sci USA 1993; 90:4971-4975.

34 Stoilov P, Castren E, Stamm S: Analysis of the human TrkB gene genomic organization reveals novel TrkB isoforms, unusual gene length, and splicing mechanism. Biochem Biophys Res Commun 2002;290:10541065.

35 Klein R, Conway D, Parada LF, Barbacid M: The TrkB tyrosine protein kinase gene codes for a second neurogenic receptor that lacks the catalytic kinase domain. Cell 1990;61:647-656.

-36 Luberg K, Wong J, Weickert CS, Timmusk T: Human TrkB gene: novel alternative transcripts, protein isoforms and expression pattern in the prefrontal cerebral cortex during postnatal development. J Neurochem 2010; 113:952-964. 